\title{
Cancer-induced cardiac cachexia: Pathogenesis and impact of physical activity (Review)
}

\author{
YASSINE BELLOUM $^{1}$, FRANÇOISE RANNOU-BEKONO ${ }^{2}$ and FRANÇOIS B. FAVIER ${ }^{1}$ \\ ${ }^{1}$ DMEM, INRA, Univ. Montpellier, Montpellier 34060; ${ }^{2}$ EA 1274, Laboratoire 'Mouvement, \\ Sport, Santé', Université de Rennes 2-ENS Rennes, Bruz 35170, France
}

Received September 22, 2016; Accepted January 30, 2017

DOI: $10.3892 /$ or.2017.5542

\begin{abstract}
Cachexia is a wasting syndrome observed in many patients suffering from several chronic diseases including cancer. In addition to the progressive loss of skeletal muscle mass, cancer cachexia results in cardiac function impairment. During the severe stage of the disease, patients as well as animals bearing cancer cells display cardiac atrophy. Cardiac energy metabolism is also impeded with disruption of mitochondrial homeostasis and reduced oxidative capacity, although the available data remain equivocal. The release of inflammatory cytokines by tumor is a key mechanism in the initiation of heart failure. Oxidative stress, which results from the combination of chemotherapy, inadequate antioxidant consumption and chronic inflammation, will further foster heart failure. Protein catabolism is due to the concomitant activation of proteolytic systems and inhibition of protein synthesis, both processes being triggered by the deactivation of the Akt/mammalian target of rapamycin pathway. The reduction in oxidative capacity involves AMP-activated protein kinase and peroxisome proliferator-activated receptor gamma coactivator $1 \alpha$ dysregulation. The nuclear factor- $\kappa \mathrm{B}$ transcription factor plays a prominent role in the coordination of these alterations. Physical exercise appears as an interesting non-pharmaceutical way to counteract cancer cachexia-induced-heart failure. Indeed, aerobic training has anti-inflammatory effects, increases anti-oxidant defenses, prevents atrophy and promotes oxidative metabolism. The present review points out the importance of better understanding the concurrent structural and metabolic changes within the myocardium during cancer and the protective effects of exercise against cardiac cachexia.
\end{abstract}

Correspondence to: Dr François B. Favier, DMEM, INRA, Univ. Montpellier, 2 place Pierre Viala, Bâtiment 22, Montpellier 34060, France

E-mail: francois.favier@umontpellier.fr

Key words: heart, exercise, atrophy, inflammation, oxidative stress, insulin resistance

\section{Contents}

1. Introduction

2. Phenotype of cardiac remodeling during cancer

3. Mechanisms initiating cardiac cachexia during cancer

4. Molecular mechanisms underlying cardiac cachexia during cancer

5. Positive effects of physical exercise on cardiac cachexia

6. Conclusions

\section{Introduction}

Thirty to $80 \%$ of cancer patients display a cachectic state mostly attributed to involuntary weight loss greater than $5 \%$ after 6 months of diagnosis $(1,2)$. This complication is responsible for a decreased quality of life, a reduced tolerance to therapy (3) and for more than $20 \%$ of death in cancer patients (4). Cancer cachexia is a complex clinical syndrome with progressive development from pre-cachexia stage to refractory cachexia (2). Clinically, cancer cachexia leads to a severe loss of fat and skeletal muscle mass usually associated with anorexia, anemia and a pronounced asthenia with considerable alterations of lipid and protein metabolism (2-4). Cancer cachexia is not fully explained by caloric restriction. Although it can have a key role in muscle loss, it remains elusive whether caloric restriction is responsible for the genesis of cancer cachexia. Indeed, the mechanisms of muscle loss and metabolic profiles are distinct between tumor-bearing and long-term fasting subjects (5). Furthermore, nutritional supplementation and pharmacological manipulation of appetite are unable to restore loss of lean body mass $(4,6)$. It has been proposed that skeletal muscle is the major tissue involved in cancer cachexia, accounting for $40 \%$ of total body weight loss $(3,6)$. However, other recent studies suggest that other tissues such as brain, liver and heart could also be implicated in the cachectic processes and directly impact muscle loss (7). Hence, cancer cachexia should be considered as a multifactorial syndrome.

Numerous studies managed to characterize mechanisms underlying skeletal muscle atrophy during cancer. However, little attention has been paid to the alteration of myocardial structure and metabolic disorders that occur in the heart. It was proposed in the 1970's that $7 \%$ of death during cancer 
could be attributed to heart failure (8), an assumption supported by the reduction in cardiac mass and function in patients (9). Independently from cancer itself, anticancer treatment including chemo- and/or radiotherapies may have some deleterious effects on myocardium and can thus exacerbate cardiac impairments that occur during cancer cachexia $(10,11)$. Patients being treated for a chronic illness like cancer were often told by their doctor to rest and reduce their physical activity. For a long time, exercise was thought to cause pain, high heart rate, dyspnea and fatigue. In contrast, randomized controlled trials showed a beneficial effect of physical activity on physical fitness, quality of life, anxiety or self-esteem, and exercise is now thought as a counter-measure that could attenuate cardiac cachexia in patients (12).

Given the importance of cardiac function on health, it is crucial to identify the molecular basis of cancer-induced cardiac cachexia and to propose alternative tools for its prevention. The present review aims to characterize cardiac remodeling and to depict the potential benefits of physical exercise on heart function during cancer cachexia.

\section{Phenotype of cardiac remodeling during cancer}

Structural and functional aspects. Ectopic implantation of C26 colon carcinoma cells is a widely used model of cancer cachexia in rodent. Myocardial atrophy is a common feature observed in this model (13-15) with a decrease in heart weight reaching $20 \%$ in tumor-bearing mice (Table I). This atrophy seems to be greater in males compared to females (-22 vs. $-10 \%$ in heart weight 27 days after tumor inoculation) (15). This striking difference in myocardial atrophy could be explained by sexual hormones as inhibition of estrogens receptor signaling, totally abolished this protective mechanism (15). The mode of administration can also influence the severity of atrophy, a more pronounced decrease in heart weight being recorded after intraperitoneal vs. subcutaneous injection of C26 tumor cells (16). Importantly, cardiac atrophy is not restricted to C26 model as it has been observed in several animal models including cancer cell inoculation, genetic models and chemically-induced tumorigenesis (Table I). Moreover, reduced heart mass has been reported in cachectic patients (gastrointestinal, pancreatic and non-small cell lung cancer) compared with non-cachectic cancer patients and controls (17).

Echocardiographic measures confirm the loss of cardiac mass since a decrease in left ventricle (LV) posterior wall thickness was observed in many studies (Table I). Tian et al (18) also reported a $28 \%$ decrease in interventricular septum along with a $30 \%$ decrease of LV posterior wall thickness. Atrophy of cardiac wall is related to a reduction of cardiomyocyte cross sectional area (14,19-21). Moreover, electronic microscopy revealed disturbed alignment of sarcomeric arrangement further demonstrating the impairment of myocardium muscle structure with cancer (13). De novo expression of fetal genes is commonly observed in studies underlying mechanisms of cardiac remodeling including cancer cachexia. For example, strong re-expression of myosin heavy chain (MyHC)- $\beta$ was recorded in $\mathrm{C} 26$ tumorbearing mice at the expense of MyHC- $\alpha(13,15,18)$. In line with this, other contractile protein such as troponin I, myosin light chain, $\alpha$-actin are degraded $(13,15)$. Accordingly, this degradation of contractile protein could deleteriously impact cardiac strength generation (22).

Besides, histological analyses show a potent fibrosis as $50-65 \%$ of fibrosis was found in heart of C26 tumor bearing mice $(13,15)$. Fibrosis of cardiac tissue is usually implicated in myocardial pathology and contributes to decrease heart performance. This could be explained by the deposit of collagen causing heart stiffness, hence the alteration of cardiac function. In fact, fibrosis can induce heart failure (HF) through the fading of diastolic function (23).

Altogether, these data unveil the potential impairment of myocardium structure during cancer cachexia which is essentially represented by a high fibrosis, an impaired sarcomeric structure and a decrease in wall cardiac thickness. These morphological abnormalities will negatively impact cardiac function (Table I). For example, Cosper and Leinwand (15) in 2011 reported a $30 \%$ reduction in aortic pressure and a $16 \%$ decrease in aortic velocity in tumor-bearing mice compared to controls. Impaired fraction ejection and fractional shortening have also been observed in $\mathrm{C} 26$ and $\mathrm{AH}-130$ tumor bearing animals (Table I). Moreover, MyHC- $\beta$ is associated with a lower ATPase activity compared to $\alpha$ isoform and re-expression of the fetal MyHC- $\beta$ is therefore associated with altered LV function (24).

In summary, along with morphological and functional abnormalities observed during cancer cachexia, all the data exposed above provide evidence claiming that cancer cachexia will ultimately lead to HF. In fact, HF would be a slow mechanism related to cancer cachexia and could be prominent before death. In turn, HF will accelerate cachexia (11). It is hypothesized that myocardial energetic perturbation that occurs during HF is due to depletion of ATP with impaired efficiency of mechanical work leading to increased resting energy expenditure (25). These data underline the importance of investigating myocardial energy balance during cancermediated cardiac cachexia.

Metabolic aspects. It is well established that alteration of energy balance contributes to cancer cachexia, increased energy expenditure being the main cause of wasting associated with cachexia (33). Indeed, a study conducted on a group of 297 unselected cancer patients showed that $49 \%$ of patients developed a hyper metabolism (34) and similar conclusion was already drawn earlier (35). Abnormalities in carbohydrate and lipid metabolism and/or mitochondrial dysfunction are major biochemical bases of elevated resting energy expenditure. Mitochondria within the myocardium represent $30 \%$ of the cardiac volume (36). In adult mammalian myocardium, ATP production is essentially mediated by mitochondrial oxidative phosphorylation as 70\% of ATP is derived from fatty acid oxidation while the rest is provided by glucose and lactate oxidation along with other ketone body (37). The capacity of heart to oxidize fatty acids is a key feature as an alteration of lipid metabolism is classically associated to HF (38).

Early report evidenced a decrease in the total mitochondrial volume and in citrate synthase activity (a marker of mitochondrial content) within hearts of methylcholanthreneinduced sarcoma mice (20). In addition, Tian et al (13) showed impaired mitochondrial structures in heart of tumor-bearing mice, including broken membranes and disorganized cristae. 
Table I. Structural and functional alterations of cardiac muscle during cancer cachexia.



$\mathrm{Apc}^{\mathrm{min} /+}$, adenomatous polyposis coli muted mice; $\mathrm{CO}$, cardiac output; $\mathrm{EF}$, ejection fraction; FS, fractional shortening; HW, heart weight; LV, left ventricle; LVSD LV, systolic diameter; M, mouse; MCA, methylcholanthrene; PWT, posterior wall thickness; R, rat; SV, stroke volume. -, + and $=$, respectively mean: significant decrease, significant increase or no significant change for the corresponding parameter.

These alterations can result in a decreased capacity to oxidize energetic substrates. Although they did not report significant alteration in mitochondrial structure, Muhlfeld et al (30) observed an increase in triglyceride content which is consistent with an impaired capacity of lipid oxidation. However, others found no change (20) or even a decrease in lipid content (39). Among the few data available, results from Drott and coworkers show an increase in cardiac oxygen consumption together with a decrease in glucose uptake in non-cachectic tumor-bearing rats $(39,40)$.
Glucose entry into cardiac cells is enhanced by insulin which triggers glucose transporter type 4 (GLUT4) translocation to the membrane. A decrease in insulin sensitivity can thus explain the reduction in glucose uptake observed in these rats. In addition, upregulation of GLUT1 expression concomitantly to a decrease in GLUT4 expression has been observed in tumor-bearing mice (18). GLUT1 expression is usually restricted to fetal tissue and is responsible for a lower level of glucose transport compared to the GLUT4 isoform (41). Re-expression of GLUT1 could be an adaptive response of the 
organism in order to compensate hypoglycemia that can occur at the latest stage of cancer, although the issue of heart metabolism during cancer cachexia needs to be better characterized.

Overall, these alterations could explain the morphological and functional abnormalities in myocardium of tumor-bearing mice, especially the adenocarcinoma C26 model. Regardless of tumor model, however, few studies have investigated the extended myocardium atrophy including functional implications and signaling pathways.

\section{Mechanisms initiating cardiac cachexia during cancer}

Inflammation. Heart metabolism can be altered by chronic low grade inflammation. In the C26 tumor model, pro-inflammatory cytokines are released by the tumor and this process is believed to promote cancer cachexia (42). The pro-inflammatory interleukin-6 (IL-6) is strongly induced in heart from cancer mice $(13-15,18)$. If acute cytokines release can protect tissues from insult, chronic elevation in pro-inflammatory mediators fosters heart dysfunction. Indeed, elevated IL-6 levels are associated with LV dysfunction (43) and it has been proposed that IL- 6 contributes to the induction of fetal genes such as MyHC- $\beta$ (44).

Similarly to IL-6, tumor necrosis factor- $\alpha$ (TNF- $\alpha$ ), another key mediator of inflammatory response, is involved in the physiopathology of diverse cardiomyopathies (45). TNF- $\alpha$ is notably a potent activator of MuRF1, an E3 ligase involved in protein breakdown (cf. below), and can thus promote muscle atrophy (46). Accordingly, an early study reported that inhibition of TNF- $\alpha$ slowed down skeletal and cardiac proteolysis in rats bearing the Yoshida AH-130 hepatoma (47).

Furthermore, Fn14, the cognate receptor of the cytokine TNF-like weak inducer of apoptosis (TWEAK), is highly expressed by $\mathrm{C} 26$ tumor cells and tumor-secreted Fn14 was demonstrated to initiate cachexia (48). In fact, treatment with Fn14 antibody but not genetic knockdown of Fn14 or TWEAK in host slowed tumor growth and extended lifespan of tumorbearing C26 mice. Additionally, myocardium atrophy was prevented in cancer cell-injected animals treated with antiFn14. Although TWEAK/Fn14 promotes inflammation via activation of the nuclear factor-kappa B (NF- $\mathrm{kB}$ ), it was not established whether the protective effects of Fn14 inhibition were related to modulation of inflammatory response.

Recently, seven tumor-secreted factors by $\mathrm{C} 26$ cells have been identified to trigger cardiomyocytes atrophy and aberrant lipid metabolism (21). Among these so-called 'cachexokines', elevated serum Ataxin-10 is a common feature of several colon tumor secretomes and is also highly expressed in pancreatic cancer and in mouse model of obesity and type II diabetes. Furthermore, serum Ataxin-10 expression correlates with body wasting level. Altogether, these findings led the authors to propose serum Ataxin-10 as a potential marker of cardiac cachexia. Besides, deleterious effects of prolonged inflammation are also related to the increase in oxidative stress.

Oxidative stress. Signs of oxidative stress have been observed in heart of rats inoculated either with Walker-256 tumors (32) or with AH-130 Yoshida hepatoma cells (49). In this model, it was demonstrated that proteins involved in muscle contraction and metabolism are significantly more oxidized in heart of the cachectic animals (14). Moreover, administration of resveratrol, a potent antioxidant, prevented cardiac atrophy in C26 tumor-bearing mice. Surprisingly, positive effect of resveratrol on skeletal muscle mass was not retrieved in LLC or AH-130 tumor-bearing animals, suggesting cancer-specific effects (50).

Mitochondrion is a major source of reactive oxygen species (ROS). In addition to inflammation, abnormality in respiratory chain function can lead to enhanced oxidative stress. A disruption in the balance between pro- and anti-oxidant systems may also occur via impaired delivery of vitamins and antioxidants resulting from anorexia, nausea and vomiting in cancer patients (51). Lastly, anti-neoplastic drugs such as cisplatin or doxorubicin could be responsible for ROS accumulation, a condition known for promoting muscle atrophy $(52,53)$. In addition to mitochondria, xanthine oxidase can generate ROS. Accordingly, inhibition of xanthine oxidase reduces cardiac atrophy and maintains heart function in rats inoculated with AH-130 Yoshida hepatoma cells (54). This further demonstrates the role of ROS overproduction in cardiac cachexia.

ROS could alter calcium homeostasis by stimulating calcium release and inhibiting calcium re-uptake by the sarcoplasmic reticulum which result in calcium overload. Calcium overload contributes to impair contractile function by promoting release of the pro-apoptotic factor cytochrome $c$. In fact, being located near the sites of calcium efflux, mitochondria will capture excessive calcium load. This in turn results in the opening of the mitochondrial permeability transition pore and the subsequent release of cytochrome $c$ which activates apoptosis pathways. The unfavorable oxidative balance that occurs during cancer also induces apoptosis through p38 MAPK-dependent activation. Inhibitors of p38-MAPK have been shown to prevent ROS-induced apoptosis, suggesting that targeting this signaling pathway could attenuate cardiac dysfunction and decrease myocyte apoptosis (55).

Insulin resistance. Cardiac mass and metabolism are also regulated by insulin. Insulin activates protein synthesis and represses proteolysis through binding to its receptor and subsequent activation of the Akt/mammalian target of rapamycin complex 1 (mTORC1) pathway (see next section). In addition, insulin promotes GLUT4-mediated glucose uptake into cardiac cells in an Akt-dependent way. A defect in insulin sensitivity or secretion, both observed in cancer $(7,56)$, would thus impair both cardiac mass and metabolism. Chronic inflammation is an identified cause of insulin resistance in skeletal muscle but also in heart (57). Notably, insulin resistance and mitochondrial dysfunction initiate heart failure in a model of pressure overload (58), underlining the importance of metabolic features in cardiac function.

\section{Molecular mechanisms underlying cardiac cachexia during cancer}

Atrophic pathways. Cardiac muscle mass is finely controlled by the balance between protein synthesis and breakdown. The Akt/mTORC1 pathway controls the protein metabolism through the concomitant stimulation of protein translation and inhibition of proteolytic pathways. Akt is activated by insulin or insulin-like growth factor-1 (IGF-1). A decrease in 
Akt/mTORC1 pathway activation will thus result in muscle atrophy because of reduced protein synthesis and enhanced protein breakdown. Accordingly, Manne et al (26) reported a decrease in cardiac protein synthesis together with a reduction of mTORC1 activity in adenomatous polyposis coli muted mice $\left(\mathrm{Apc}^{\mathrm{Min} /+}\right)$, a model of colorectal cancer. Consistent with these findings, tumor-bearing AH-130 rats display a marked reduction in IGF-1 receptor and reduced phosphorylation of key downstream targets, including Akt and GSK3 $\beta$ (17). In contrast, Akt/mTORC1 pathway was overactivated in N-Methyl-N-nitrosourea (MNU)-induced breast cancer rats (59) and although mTORC1 activity was reduced, Akt phosphorylation was indeed increased in $\mathrm{Apc}^{\mathrm{Min} /+}$ mice (26). If sustained activity of the $\mathrm{Akt} / \mathrm{mTORC} 1$ pathway may spare cardiac mass under cancer cachexia, excessive activation can lead to pathological hypertrophy associated with fibrosis (59). This points to a complex dysregulation of the Akt/mTORC1 pathway in heart with cancer. In contrast to IGF-1, myostatin has negative effects on Akt/mTORC1 activation (60). Mammary tumorigenesis induced by administration of MNU leads to enhanced myostatin expression in the myocardium (59). Higher levels of phosphorylated Smad-3, the down-stream effector of myostatin, were also observed (59). Similar results were found in myocardium of AH-130 tumor-bearing rats (17). Notably, pharmacological blockage of the myostatin receptor reversed both skeletal and cardiac muscle wasting during cancer cachexia (29). Old myostatin KO mice display lower fibrosis and improved cardiac function (61) further underlining the potential role of myostatin in the pathogenesis of HF. However, cardiac-specific myostatin deletion did not restore either heart mass or function in a model of HF in mice (62). Therefore, the effect of myostatin inhibition on heart function during cancer remains to be ascertained.

Importantly, the Akt/mTORC1 signaling inhibits the two major pathways responsible for proteolysis: the ubiquitin/ proteasome system and the autophagy/lysosome process. Proteasomal degradation requires the labelling of targeted proteins through the successive activity of the so-called ubiquitin-activating (E1), -conjugating (E2) and -ligase (E3) enzymes. Polyubiquitinylated proteins are then addressed to and hydrolyzed by the $26 \mathrm{~S}$ proteasome. Two muscle-specific E3 enzymes, Atrogin-1 (also called MAFbx or Fbox32) and muscle ring finger 1 (MuRF1), have been widely studied because of their crucial role in the regulation of both skeletal and cardiac muscle mass. Indeed, MuRF1 KO mice are protected against cardiac atrophy induced by glucocorticoids or by the reversal of transaortic constriction (63) and MuRF1 transgenic mice have thinner LV wall thickness (64). Atrogin-1 has been shown to inhibit cardiac hypertrophy, notably through the degradation of calcineurin (65) while its adenoviral overexpression reduces cardiomyocytes size (66). Consistently, increased cardiac expression of MuRF1 and Atrogin-1 has been reported in mice inoculated with colon-26 adenocarcinoma cells $(16,18,19)$ or colon adenocarcinoma cell lines 16 (31). Interestingly, Atrogin-1 expression is also increased in response to doxorubicin, an effective antitumor agent (66). MuRF1 targets structural proteins such as MyHC or troponin 1 (reviewed in ref. 67). However, recent advances point out the role of MuRF1 in energetic metabolism, notably through the degradation of the creatine kinase. There are few data on the role of other E3 ligases (e.g. F-box and leucine-rich repeat protein 22 , murine double minute 2 or c-terminus of heat shock protein 70 -interacting protein) in cancer. Therefore, one may suppose that the knowledge about the implication of the ubiquitin/proteasome system in cardiac remodeling during cancer cachexia is still fragmented.

Autophagy (ATG) is a process that ensures the degradation of long-lived proteins, macromolecules and organelles including lipids, glycogen, mitochondria or ribosomes by lysosomal vesicles. In skeletal or cardiac muscle, basal ATG is important to recycle damaged-mitochondria and to provide amino acids for protein synthesis. Excessive activation or inhibition of ATG flux contributes to muscle atrophy (68) and to the development of heart disease (69). An increase in the expression of LC3, which controls a key step in ATG initiation, and of cathepsin L, a lysosomal protease, has been observed in the hearts of C26 tumor-bearing mice (15). Importantly, these animals displayed strong increase in the protein content of activated LC3 and atrophic hearts have increased autolysosomes and autophagosomes number. Manne et al (26) also observed an increase in Beclin protein expression, another marker of ATG activation. Recently, restoration of cardiac mass and function in tumor-bearing rats by appetite stimulation was associated with the normalization of ATG markers (70). This emerging literature reinforces the idea that ATG contributes to cardiac muscle atrophy with cancer.

ATG and ubiquitin/proteasome systems can be transcriptionally activated by the forkhead family of transcription factors FOXO1, FOXO3A and FOXO4 in skeletal muscle (71). FOXO is directly phosphorylated by Akt, leading to its cytosolic sequestration. FOXOs inhibition partly prevented skeletal muscle atrophy following C26 cells inoculation (72). In heart, FOXO3A promotes MuRF1 and Atrogin-1 expression while it inhibits cardiomyocyte hypertrophy and reduces cardiac cell size (73). Therefore, FOXOs could be considered as a key regulator of cardiac atrophy in tumor-bearing mice. However, while there is strong presumption about the implication of FOXOs in heart atrophy during cancer cachexia, further work remains to be done in order to characterize its role.

$\mathrm{NF}-\kappa \mathrm{B}$ is another central transcription factor involved in cardiac cachexia. NF- $\kappa \mathrm{B}$ exists as a heterodimer of two subunits (p65 and p50), which are sequestered in an inactive form in the cytoplasm by the repressor $\mathrm{I} \kappa \mathrm{B} \alpha$. Inhibition of $\mathrm{NF}-\kappa \mathrm{B}$ prevents skeletal muscle loss under denervation and cancer cachexia (74). NF- $\kappa \mathrm{B}$ acts notably through MuRF1 to promote muscle atrophy $(74,75)$. NF- $\kappa \mathrm{B}$ expression is upregulated in the heart of tumor-bearing animals $(14,59)$. Resveratrol inhibits I $\mathrm{B}$ kinase-mediated $\mathrm{NF}-\kappa \mathrm{B}$ activation, and promotes SIRT1-dependent p65 deacetylation, resulting in the repression of NF- $\kappa \mathrm{B}$ transcriptional activity (14). Resveratrol also blunted MuRF1 expression (14) and prevented cardiomyocyte atrophy as well as functional impairment in mice implanted with C26 cancer cells (19). Importantly these beneficial effects occurred without alteration of tumor-derived cytokines release underlining the direct effect of $\mathrm{NF}-\kappa \mathrm{B}$ on cardiac cells.

Oxidative metabolism. Cardiac ATP production is highly dependent on lipid metabolism. Lipid oxidation is transcriptionally promoted by the peroxisome-proliferator activated receptor $\alpha(\operatorname{PPAR} \alpha)$. Among the genes upregulated by PPAR $\alpha$, 
CPT1 (carnitine palmitoyltransferase) is a critical step of fatty acid $\beta$ oxidation as it allows the entry of fatty acid in mitochondria. At a refractory stage, cardiac PPAR $\alpha$ and CPT1 mRNA expressions were significantly reduced in C26 tumor-bearing mice (18). Similar results were observed using unloaded hearts that exhibit cardiomyocyte atrophy (76). As stated before, this metabolic reshuffle from lipid to glucose is harmful to cardiac metabolism as it has been associated to imminent HF occurrence (77). A greater glucose oxidation can nevertheless reflect a positive adaptation. Indeed, glucose oxidation provides more energy (ATP) per molecule of oxygen consumed compared to fatty acid (higher $\mathrm{P} / \mathrm{O}$ ratio). However, if insufficiently compensated, the disturbance of energy balance capacity will impede heart function. In physiological conditions, AMPK plays a crucial role in protecting cardiac cells from energy homeostasis perturbations via activation of catabolic pathways to generate ATP. ROS can reduce AMPK activation by oxidation of cysteine residues (78), leading to decreased phosphorylation of ACC (acetyl-CoA carboxylase), an AMPK downstream target. The lack of ACC inhibition will then result in the impairment of CPT1-dependent fatty acid entry into the mitochondria.

A TNF- $\alpha / \mathrm{NF}-\kappa \mathrm{B}$ pathway has been proposed to increase glucose oxidation at the expense of lipids, by the inhibition of PPAR $\gamma$ coactivator $1-\alpha$ (PGC-1 $\alpha$ ) (79). In addition, although its use has been restricted due to cardiac complications, the PPAR $\gamma$ agonist rosiglitazone has been shown to improve the phenotype of AH-130 tumor-bearing rats (28). Indeed, treatment restored several markers of cardiac function, increased septum thickness together with the abolition of proteasome enzymatic activity upregulation and enhanced locomotor activity. Rosiglitazone has anti-diabetic effects via the improvement of insulin sensitivity, and PPAR $\gamma$ activation has also anti-inflammatory actions on cardiovascular system notably by the inhibition of NF- $\mathrm{BB}$ (reviewed in ref. 80). However, these results should be interpreted with cautious because of differences in PPAR $\gamma$ agonists effects between mouse models and human (80). Cardiomyocytes cultured with conditioned medium from $\mathrm{C} 26$ cells display aberrant fatty acid oxidation (21), suggesting that tumor released-factors will not systematically inhibit lipid oxidation as previously suggested by Drott and coworkers. Altogether this points to the importance of better understanding lipid metabolism regulation in heart during cancer. Interestingly, PPARs and AMPK could be major targets for an effective strategy to offset the altered cardiac metabolism.

All the mechanisms described above highlight the importance of decreasing inflammation, oxidative stress and insulin resistance in order to maintain: i) cardiac muscle mass; ii) mitochondrial homeostasis; and iii) oxidative capacity (Fig. 1).

\section{Positive effects of physical exercise on cardiac cachexia}

A number of studies confirm the beneficial effects of regular aerobic exercise on heart function in pathological conditions (reviewed in ref. 81). For instance, hypertensive rats displayed significant reduction of cardiac fibrosis (40\%) upon aerobic training and this was associated with improved diastolic function (82). Similarly, endurance training reduced myocardial infarction-mediated fibrosis (83). Endurance training is also



Figure 1. The main mechanisms leading to heart failure during cancer cachexia. Combined effects of inflammation and oxidative stress stimulate muscle atrophy, reduction in oxidative capacity and mitochondrial dysfunction. Activation of NF- $\kappa \mathrm{B}$ increases ubiquitin/proteasome-dependent proteolysis and contributes to decrease activity of PGC-1 $\alpha$, a key transcription factor involved in the upregulation of the oxidative metabolism. Calcium overload will promote cytochrome c release by the mitochondria, an event known for initiating apoptosis. In time, all these impairments will then lead to heart failure.

responsible for physiological hypertrophy in cardiac myocytes and improves contractile function as attested by higher calcium sensitivity and enhanced calcium handling in female rats compared to sedentary (84). In physiological conditions, aerobic exercise increases MyHC- $\alpha$ protein expression while $\beta$ isoform content remains unchanged $(85,86)$. This mechanism also occurs in pathological conditions since exercise prevented the increase in MyHC- $\beta$ to MyHC- $\alpha$ mRNA ratio after myocardial infarction (83). In cancer, aerobic training restores the tolerance to physical activity allowing therefore, a better quality of life (87). Importantly, regular physical activity seems to slow down tumor growth (88). This finding is confirmed by Goh et al (89), which unveiled the potent role of exercise training as a preventive measure for tumor progression. In fact, results of this study show that the longer distance tumorbearing mice browse, the smaller their tumor is. Furthermore, it has been shown that aerobic training enhances life span of rats bearing Walker 256 tumor (90).

Exercise and systemic inflammation. Aerobic training has been shown to reduce chronical inflammation (87), although acute endurance exercise increases serum IL- 6 and myocardial IL-6 receptor protein content (91). Noteworthy, cytokines response to exercise differs from the ones that occur in chronic diseases characterized by an inflammatory state (92). Indeed, plasmatic TNF- $\alpha$ and IL-1 $\beta$ (two pro-inflammatory cytokines) do not increase in response to exercise. Exercise is also associated with high levels of anti-inflammatory cytokines like IL-10 and pro-inflammatory cytokines inhibitors such as IL-1ra (antagonist receptor for IL-1), sTNF $\alpha$-r1 and sTNF $\alpha-r 2$ (antagonist receptors for TNF- $\alpha$ ). Accordingly, it has recently been shown that endurance exercise blunts TNF- $\alpha$-mediated NF- $\mathrm{KB}$ activation in skeletal muscle (93) and regular exercise 
limits TNF- $\alpha$, IL-6 and IL-1 $\beta$ induction in heart after myocardial infarction (83). During multiple pathologies, the stress is chronic with low and constant level of pro-inflammatory cytokines as observed for IL-6 during C26-induced cardiac cachexia (15). Exercise will modulate this low grade of systemic inflammation by establishing an anti-inflammatory environment via anti-inflammatory cytokines release (IL-1ra and IL-10) and cytokines inhibitors (sTNF $\alpha-r 1$ and sTNF $\alpha-r 2)$. This releasing process, initiated within local tissues and progresses to peripheral ones, is essentially mediated by the myokine IL-6 underlining its anti-inflammatory effect when induced by exercise (92). In agreement, a recent report showed that 35 weeks of aerobic training prevented the increase in TWEAK expression (in serum and heart) as well as NF- $\mathrm{BB}$ activation but did not alter myostatin expression in C26 tumorbearing mice (59). This result suggests that moderate exercise training could modulate cancer-induced cardiac remodeling through the TWEAK/NF- $\mathrm{B}$ signaling.

Oxidative stress and exercise. The beneficial effect of aerobic training on redox status is well documented (94) and the protective effects of physical activity against prostate cancer have been proposed to be related to enhancement of antioxidant system (95). In heart, regular physical activity at a low intensity enhances the expression of anti-oxidant enzymes such as Mn-superoxide dismutase (SOD) and $\mathrm{Cu} / \mathrm{Zn}-\mathrm{SOD}$, glutathione peroxidase 1 and catalase (55). Importantly, Mn-SOD overexpression is essential to integral heart protection against ischemia/reperfusion, a condition known for inducing ROS. In agreement, doxorubicin-mediated impairment in LV function and LV lipid peroxidation, a sign of oxidative stress, were attenuated with exercise (96).

Exercise also induces the expression of heat shock protein (HSP) 60 and 72 that may contribute to myocardial protection from ROS. The putative mechanisms by which HSP can protect cardiomyocytes include control of protein folding, prevention of denaturation and aggregation of intracellular proteins, and acceleration of the breakdown of damaged proteins. HSP72 seems to be involved in cellular protection against a variety of stresses (97). Importantly, exercise reduces cell death via modulation of pro- and anti-apoptotic gene expression (81) and HSP are thought to protect against apoptosis. For instance, the HSP70 overexpression protected against ischemia-induced tissue damage and would be associated to enhanced cell survival (98). Altogether, these data are encouraging enough to consider aerobic exercise as a promoting therapeutic target to prevent and counteract ROS-induced cardiac dysfunction (55).

Oxidative metabolism. Endurance capacity is significantly reduced during cancer cachexia. This functional impairment has been associated to mitochondrial dysfunction in skeletal muscle and myocardium (99). In physiological conditions, aerobic training is able to stimulate oxidative metabolism and insulin sensitivity (87). Indeed, 6 weeks of endurance training enhanced mitochondrial protein content from 50 to $100 \%$ in hearts (100), an observation corroborated by microarray analysis (85). Mitochondrial biogenesis is notably driven by exercise-induced PGC-1 $\alpha$ upregulation $(100,101)$. Activation of the PGC1- $\alpha$-PPAR complex with endurance training logically results in enhanced fatty acid oxidation and could

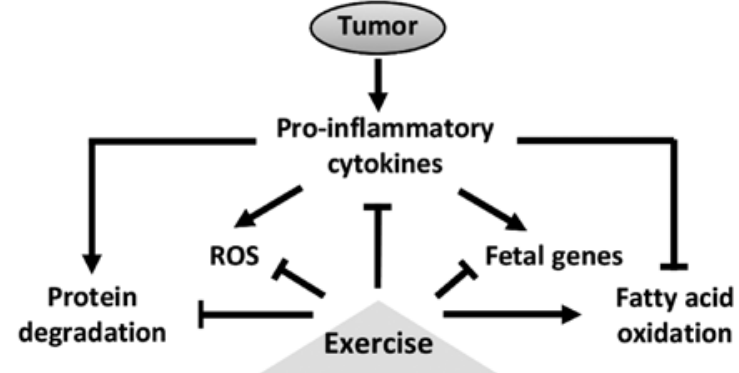

Figure 2. Protective effects of exercise on cancer cachexia-induced mechanisms of heart failure. Chronic aerobic exercise antagonizes most negative effects of tumor-derived cytokines on cardiac muscle such as increased proteolysis, enhanced reactive oxygen species (ROS) production, re-expression of fetal genes and impaired fatty acid oxidation. In addition endurance exercise has been demonstrated to have anti-inflammatory effects pointing to the interest of physical activity in chronic diseases including cancer.

be associated to oxidative profile switch $(100,102)$. AMPK activation also results in facilitation of fatty acid oxidation (cf. above) and exercise is a potent activator of AMPK activity in cardiac tissue (103). Furthermore, GLUT4 translocation and expression is enhanced ensuing aerobic training in myocardium of sedentary subjects (104). Therefore, aerobic training would prevent or restore cardiac lipid oxidation during cancer cachexia through activation of AMPK and PPAR/PGC-1 $\alpha$ signaling.

IGF-1/Akt signaling and heart mass with exercise. IGF-1/ $\mathrm{PI} 3 \mathrm{~K} / \mathrm{Akt}$ signaling pathway is involved during physiological hypertrophy of cardiomyocytes in response to aerobic training. For example, six weeks of aerobic training induce physiological cardiac hypertrophy (85) and this response was blunted in KO Akt mice after a 3 weeks swimming program (105). Importantly, hearts overexpressing IGF-1 receptor displayed PI3K-dependent cardiac hypertrophy with no evidence of histopathology (106). Activation of the IGF-1/PI3K pathway seems to be beneficial in pathogical models. Indeed, overexpressing PI3K in load-pressure model mice reduced interstitial fibrosis and attenuated pathological growth (107). Cardiacspecific IGF-1 overexpression enhanced myocardial function and cell reparation after cardiac infarction (108). Altogether, these data support the idea that aerobic training could delay the onset and/or prevent the progression of cardiac dysfunction during cancer via enhanced IGF-1/PI3K activity.

\section{Conclusions}

Understanding cancer physiopathology is a huge challenge, notably because of the multiple types of cancers. However, cachexia is a common feature of the disease at advanced stage. This devastating syndrome induces HF, which in turn exacerbates cachexia and worsens vital prognosis. The exact sequence of events leading to cancer-related HF still remains to be established. Cardiac muscle catabolism results from an imbalance between energy expenditure and capacity production, and this perturbation is related to inflammation and oxidative stress. Exercise is a powerful antidote against such mechanisms and can therefore protect myocardium from cancer itself and therapies side effects (Fig. 2). However, the 
modalities of exercise have to be adapted to fragile population. Physical exercise is an interesting therapy as it can have both protective but also preventive effects, so it is probably best to start training as soon as possible.

\section{References}

1. Inui A: Cancer anorexia-cachexia syndrome: Current issues in research and management. CA Cancer J Clin 52: 72-91, 2002.

2. Fearon K, Strasser F, Anker SD, Bosaeus I, Bruera E, Fainsinger RL, Jatoi A, Loprinzi C, MacDonald N, Mantovani G, et al: Definition and classification of cancer cachexia: An international consensus. Lancet Oncol 12: 489-495, 2011.

3. Argilés JM, Moore-Carrasco R, Fuster G, Busquets S and LópezSoriano FJ: Cancer cachexia: The molecular mechanisms. Int J Biochem Cell Biol 35: 405-409, 2003.

4. Tisdale MJ: Cachexia in cancer patients. Nat Rev Cancer 2: 862-871, 2002.

5. Costelli P and Baccino FM: Cancer cachexia: From experimental models to patient management. Curr Opin Clin Nutr Metab Care 3: $177-181,2000$

6. Tisdale MJ: Mechanisms of cancer cachexia. Physiol Rev 89: 381-410, 2009.

7. Argilés JM, Busquets S, Stemmler B and López-Soriano FJ: Cancer cachexia: Understanding the molecular basis. Nat Rev Cancer 14: 754-762, 2014.

8. Ambrus JL, Ambrus CM, Mink IB and Pickren JW: Causes of death in cancer patients. J Med 6: 61-64, 1975.

9. Burch GE, Phillips JH and Ansari A: The cachetic heart. A clinico-pathologic, electrocardiographic and roentgenographic entity. Dis Chest 54: 403-409, 1968.

10. Ewer MS and Ewer SM: Cardiotoxicity of anticancer treatments. Nat Rev Cardiol 12: 547-558, 2015.

11. Kazemi-Bajestani SM, Becher H, Fassbender K, Chu Q and Baracos VE: Concurrent evolution of cancer cachexia and heart failure: Bilateral effects exist. J Cachexia Sarcopenia Muscle 5: 95-104, 2014

12. Murphy KT: The pathogenesis and treatment of cardiac atrophy in cancer cachexia. Am J Physiol Heart Circ Physiol 310: H466-H477, 2016.

13. Tian M, Nishijima Y, Asp ML, Stout MB, Reiser PJ and Belury MA: Cardiac alterations in cancer-induced cachexia in mice. Int J Oncol 37: 347-353, 2010.

14. Shadfar S, Couch ME, McKinney KA, Weinstein LJ, Yin X, Rodríguez JE, Guttridge DC and Willis M: Oral resveratrol therapy inhibits cancer-induced skeletal muscle and cardiac atrophy in vivo. Nutr Cancer 63: 749-762, 2011.

15. Cosper PF and Leinwand LA: Cancer causes cardiac atrophy and autophagy in a sexually dimorphic manner. Cancer Res 71: $1710-1720,2011$.

16. Matsuyama T, Ishikawa T, Okayama T, Oka K, Adachi S, Mizushima K, Kimura R, Okajima M, Sakai H, Sakamoto N, et al: Tumor inoculation site affects the development of cancer cachexia and muscle wasting. Int J Cancer 137: 2558-2565, 2015.

17. Springer J, Tschirner A, Haghikia A, von Haehling S, Lal H, Grzesiak A, Kaschina E, Palus S, Pötsch M, von Websky K, et al: Prevention of liver cancer cachexia-induced cardiac wasting and heart failure. Eur Heart J 35: 932-941, 2014.

18. Tian M, Asp ML, Nishijima Y and Belury MA: Evidence for cardiac atrophic remodeling in cancer-induced cachexia in mice. Int J Oncol 39: 1321-1326, 2011.

19. Wysong A, Couch M, Shadfar S, Li L, Rodriguez JE, Asher S, Yin X, Gore M, Baldwin A, Patterson C, et al: NF- $\kappa B$ inhibition protects against tumor-induced cardiac atrophy in vivo. Am J Pathol 178: 1059-1068, 2011.

20. Sjöström M, Wretling ML, Karlberg I, Edén E and Lundholm K: Ultrastructural changes and enzyme activities for energy production in hearts concomitant with tumor-associated malnutrition. J Surg Res 42: 304-313, 1987.

21. Schäfer M, Oeing CU, Rohm M, Baysal-Temel E, Lehmann LH, Bauer R, Volz HC, Boutros M, Sohn D, Sticht C, et al: Ataxin-10 is part of a cachexokine cocktail triggering cardiac metabolic dysfunction in cancer cachexia. Mol Metab 5: 67-78, 2015.

22. van der Velden J, Merkus D, Klarenbeek BR, James AT, Boontje NM, Dekkers DH, Stienen GJ, Lamers JM and Duncker DJ: Alterations in myofilament function contribute to left ventricular dysfunction in pigs early after myocardial infarction. Circ Res 95: e85-e95, 2004.
23. Bonne G, Carrier L, Richard P, Hainque B, Tesson F, Komajda M and Schwartz K: Génétique des cardiomyopathies hypertrophiques. Med Sci 14: 1054-1066, 1998.

24. Korte FS, Herron TJ, Rovetto MJ and McDonald KS: Power output is linearly related to MyHC content in rat skinned myocytes and isolated working hearts. Am J Physiol Heart Circ Physiol 289: H801-H812, 2005.

25. Ashrafian H, Frenneaux MP and Opie LH: Metabolic mechanisms in heart failure. Circulation 116: 434-448, 2007.

26. Manne ND, Lima M, Enos RT, Wehner P, Carson JA and Blough E: Altered cardiac muscle mTOR regulation during the progression of cancer cachexia in the Apc ${ }^{\mathrm{Min} /+}$ mouse. Int J Oncol 42: 2134-2140, 2013.

27. Palus S, von Haehling S, Flach VC, Tschirner A, Doehner W, Anker SD and Springer J: Simvastatin reduces wasting and improves cardiac function as well as outcome in experimental cancer cachexia. Int J Cardiol 168: 3412-3418, 2013.

28. Trobec K, Palus S, Tschirner A, von Haehling S, Doehner W, Lainscak M, Anker SD and Springer J: Rosiglitazone reduces body wasting and improves survival in a rat model of cancer cachexia. Nutrition 30: 1069-1075, 2014.

29. Zhou X, Wang JL, Lu J, Song Y, Kwak KS, Jiao Q, Rosenfeld R, Chen Q, Boone T, Simonet WS, et al: Reversal of cancer cachexia and muscle wasting by ActRIIB antagonism leads to prolonged survival. Cell 142: 531-543, 2010.

30. Mühlfeld C, Das SK, Heinzel FR, Schmidt A, Post H, Schauer S, Papadakis T, Kummer W and Hoefler G: Cancer induces cardiomyocyte remodeling and hypoinnervation in the left ventricle of the mouse heart. PLoS One 6: e20424, 2011.

31. Hinch EC, Sullivan-Gunn MJ, Vaughan VC, McGlynn MA and Lewandowski PA: Disruption of pro-oxidant and antioxidant systems with elevated expression of the ubiquitin proteosome system in the cachectic heart muscle of nude mice. J Cachexia Sarcopenia Muscle 4: 287-293, 2013.

32. Borges FH, Marinello PC, Cecchini AL, Blegniski FP, Guarnier FA and Cecchini R: Oxidative and proteolytic profiles of the right and left heart in a model of cancer-induced cardiac cachexia. Pathophysiology 21: 257-265, 2014.

33. Argilés JM, Fontes-Oliveira CC, Toledo M, López-Soriano FJ and Busquets S: Cachexia: A problem of energetic inefficiency. J Cachexia Sarcopenia Muscle 5: 279-286, 2014.

34. Bosaeus I, Daneryd P, Svanberg E and Lundholm K: Dietary intake and resting energy expenditure in relation to weight loss in unselected cancer patients. Int J Cancer 93: 380-383, 2001.

35. Lindmark L, Bennegård K, Edén E, Ekman L, Scherstén T, Svaninger G and Lundholm K: Resting energy expenditure in malnourished patients with and without cancer. Gastroenterology 87: 402-408, 1984.

36. Aon MA and Cortassa S: Mitochondrial network energetics in the heart. Wiley Interdiscip Rev Syst Biol Med 4: 599-613, 2012

37. Lopaschuk GD, Ussher JR, Folmes CD, Jaswal JS and Stanley WC: Myocardial fatty acid metabolism in health and disease. Physiol Rev 90: 207-258, 2010.

38. Madrazo JA and Kelly DP: The PPAR trio: Regulators of myocardial energy metabolism in health and disease. J Mol Cell Cardiol 44: 968-975, 2008.

39. Drott C, Waldenström A and Lundholm K: Cardiac sensitivity and responsiveness to beta-adrenergic stimulation in experimental cancer and undernutrition. J Mol Cell Cardiol 19: 675-683, 1987.

40. Drott $C$ and Lundholm K: Glucose uptake and amino acid metabolism in perfused hearts from tumor-bearing rats. J Surg Res 49: 62-68, 1990

41. Montel-Hagen A, Blanc L, Boyer-Clavel M, Jacquet C, Vidal M, Sitbon M and Taylor N: The Glut1 and Glut 4 glucose transporters are differentially expressed during perinatal and postnatal erythropoiesis. Blood 112: 4729-4738, 2008.

42. Yasumoto K, Mukaida N, Harada A, Kuno K, Akiyama M, Nakashima E, Fujioka N, Mai M, Kasahara T, Fujimoto-Ouchi K, et al: Molecular analysis of the cytokine network involved in cachexia in colon 26 adenocarcinoma-bearing mice. Cancer Res 55: 921-927, 1995.

43. Kanda T and Takahashi T: Interleukin- 6 and cardiovascular diseases. Jpn Heart J 45: 183-193, 2004.

44. Saito S, Aikawa R, Shiojima I, Nagai R, Yazaki Y and Komuro I Endothelin-1 induces expression of fetal genes through the interleukin-6 family of cytokines in cardiac myocytes. FEBS Lett 456: 103-107, 1999.

45. Pajak B, Orzechowska S, Pijet B, Pijet M, Pogorzelska A, Gajkowska B and Orzechowski A: Crossroads of cytokine signaling - the chase to stop muscle cachexia. J Physiol Pharmacol 59 (Suppl 9): 251-264, 2008. 
46. Bodine SC, Latres E, Baumhueter S, Lai VK, Nunez L, Clarke BA, Poueymirou WT, Panaro FJ, Na E, Dharmarajan K, et al: Identification of ubiquitin ligases required for skeletal muscle atrophy. Science 294: 1704-1708, 2001.

47. Costelli P, Carbó N, Tessitore L, Bagby GJ, Lopez-Soriano FJ, Argilés JM and Baccino FM: Tumor necrosis factor-alpha mediates changes in tissue protein turnover in a rat cancer cachexia model. J Clin Invest 92: 2783-2789, 1993.

48. Johnston AJ, Murphy KT, Jenkinson L, Laine D, Emmrich K, Faou P, Weston R, Jayatilleke KM, Schloegel J, Talbo G, et al: Targeting of Fn14 prevents cancer-induced cachexia and prolongs survival. Cell 162: 1365-1378, 2015.

49. Marin-Corral J, Fontes CC, Pascual-Guardia S, Sanchez F, Olivan M, Argilés JM, Busquets S, López-Soriano FJ and Barreiro E: Redox balance and carbonylated proteins in limb and heart muscles of cachectic rats. Antioxid Redox Signal 12: 365-380, 2010.

50. Busquets S, Fuster G, Ametller E, Olivan M, Figueras M, Costelli P, Carbó N, Argilés JM and López-Soriano FJ: Resveratrol does not ameliorate muscle wasting in different types of cancer cachexia models. Clin Nutr 26: 239-244, 2007.

51. Gould DW, Lahart I, Carmichael AR, Koutedakis Y and Metsios GS: Cancer cachexia prevention via physical exercise: Molecular mechanisms. J Cachexia Sarcopenia Muscle 4: 111-124, 2013.

52. Mantovani G, Madeddu C, Macciò A, Gramignano G, Lusso MR, Massa E, Astara G and Serpe R: Cancer-related anorexia/ cachexia syndrome and oxidative stress: An innovative approach beyond current treatment. Cancer Epidemiol Biomarkers Prev 13: $1651-1659,2004$

53. Min K, Kwon OS, Smuder AJ, Wiggs MP, Sollanek KJ, Christou DD, Yoo JK, Hwang MH, Szeto HH, Kavazis AN, et al: Increased mitochondrial emission of reactive oxygen species and calpain activation are required for doxorubicin-induced cardiac and skeletal muscle myopathy. J Physiol 593: 2017-2036, 2015.

54. Springer J, Tschirner A, Hartman K, von Haehling S, Anker SD and Doehner W: The xanthine oxidase inhibitor oxypurinol reduces cancer cachexia-induced cardiomyopathy. Int J Cardiol 168: 3527-3531, 2013.

55. Scott JM, Khakoo A, Mackey JR, Haykowsky MJ, Douglas PS and Jones LW: Modulation of anthracycline-induced cardiotoxicity by aerobic exercise in breast cancer: Current evidence and underlying mechanisms. Circulation 124: 642-650, 2011.

56. Asp ML, Tian M, Wendel AA and Belury MA: Evidence for the contribution of insulin resistance to the development of cachexia in tumor-bearing mice. Int J Cancer 126: 756-763, 2010.

57. Gray S and Kim JK: New insights into insulin resistance in the diabetic heart. Trends Endocrinol Metab 22: 394-403, 2011.

58. Zhang L, Jaswal JS, Ussher JR, Sankaralingam S, Wagg C, Zaugg $\mathrm{M}$ and Lopaschuk GD: Cardiac insulin-resistance and decreased mitochondrial energy production precede the development of systolic heart failure after pressure-overload hypertrophy. Circ Heart Fail 6: 1039-1048, 2013.

59. Padrão AI, Moreira-Gonçalves D, Oliveira PA, Teixeira C Faustino-Rocha AI, Helguero L, Vitorino R, Santos LL, Amado F, Duarte JA, et al: Endurance training prevents TWEAK but not myostatin-mediated cardiac remodelling in cancer cachexia. Arch Biochem Biophys 567: 13-21, 2015.

60. Amirouche A, Durieux AC, Banzet S, Koulmann N, Bonnefoy R, Mouret C, Bigard X, Peinnequin A and Freyssenet D: Downregulation of $\mathrm{Akt} / \mathrm{mammalian}$ target of rapamycin signaling pathway in response to myostatin overexpression in skeletal muscle. Endocrinology 150: 286-294, 2009.

61. Morissette MR, Stricker JC, Rosenberg MA, Buranasombati C, Levitan EB, Mittleman MA and Rosenzweig A: Effects of myostatin deletion in aging mice. Aging Cell 8: 573-583, 2009.

62. Heineke J, Auger-Messier M, Xu J, Sargent M, York A, Welle S and Molkentin JD: Genetic deletion of myostatin from the heart prevents skeletal muscle atrophy in heart failure. Circulation 121: 419-425, 2010.

63. Willis MS, Schisler JC, Li L, Rodríguez JE, Hilliard EG, Charles PC and Patterson C: Cardiac muscle ring finger-1 increases susceptibility to heart failure in vivo. Circ Res 105: 80-88, 2009

64. Willis MS, Rojas M, Li L, Selzman CH, Tang RH, Stansfield WE, Rodriguez JE, Glass DJ and Patterson C: Muscle ring finger 1 mediates cardiac atrophy in vivo. Am J Physiol Heart Circ Physiol 296: H997-H1006, 2009.
65. Li HH, Kedar V, Zhang C, McDonough H, Arya R, Wang DZ and Patterson C: Atrogin-1/muscle atrophy F-box inhibits calcineurin-dependent cardiac hypertrophy by participating in an SCF ubiquitin ligase complex. J Clin Invest 114: 1058-1071, 2004.

66. Yamamoto Y, Hoshino Y, Ito T, Nariai T, Mohri T, Obana M, Hayata N, Uozumi Y, Maeda M, Fujio Y, et al: Atrogin-1 ubiquitin ligase is upregulated by doxorubicin via p38-MAP kinase in cardiac myocytes. Cardiovasc Res 79: 89-96, 2008.

67. Willis MS, Bevilacqua A, Pulinilkunnil T, Kienesberger P, Tannu $\mathrm{M}$ and Patterson C: The role of ubiquitin ligases in cardiac disease. J Mol Cell Cardiol 71: 43-53, 2014.

68. Masiero E and Sandri M: Autophagy inhibition induces atrophy and myopathy in adult skeletal muscles. Autophagy 6: 307-309, 2010.

69. Levine B and Kroemer G: Autophagy in the pathogenesis of disease. Cell 132: 27-42, 2008.

70. Musolino V, Palus S, Tschirner A, Drescher C, Gliozzi M, Carresi C, Vitale C, Muscoli C, Doehner W, von Haehling S, et al: Megestrol acetate improves cardiac function in a model of cancer cachexia-induced cardiomyopathy by autophagic modulation. J Cachexia Sarcopenia Muscle 7: 555-566, 2016.

71. Milan G, Romanello V, Pescatore F, Armani A, Paik JH, Frasson L, Seydel A, Zhao J, Abraham R, Goldberg AL, et al: Regulation of autophagy and the ubiquitin-proteasome system by the FoxO transcriptional network during muscle atrophy. Nat Commun 6: 6670, 2015.

72. Judge SM, Wu CL, Beharry AW, Roberts BM, Ferreira LF, Kandarian SC and Judge AR: Genome-wide identification of FoxO-dependent gene networks in skeletal muscle during C26 cancer cachexia. BMC Cancer 14: 997, 2014.

73. Skurk C, Izumiya Y, Maatz H, Razeghi P, Shiojima I, Sandri M, Sato K, Zeng L, Schiekofer S, Pimentel D, et al: The FOXO3a transcription factor regulates cardiac myocyte size downstream of AKT signaling. J Biol Chem 280: 20814-20823, 2005.

74. Cai D, Frantz JD, Tawa NE Jr, Melendez PA, Oh BC, Lidov HG, Hasselgren PO, Frontera WR, Lee J, Glass DJ, et al: IKKbeta/ NF-kappaB activation causes severe muscle wasting in mice. Cell 119: 285-298, 2004.

75. Li H, Malhotra S and Kumar A: Nuclear factor-kappa B signaling in skeletal muscle atrophy. J Mol Med (Berl) 86: 1113-1126, 2008

76. Razeghi P, Wang ME, Youker KA, Golfman L, Stepkowski S and Taegtmeyer H: Lack of NF-kappaB1 (p105/p50) attenuates unloading-induced downregulation of PPARalpha and PPARalpha-regulated gene expression in rodent heart. Cardiovasc Res 74: 133-139, 2007.

77. Sack MN, Rader TA, Park S, Bastin J, McCune SA and Kelly DP: Fatty acid oxidation enzyme gene expression is downregulated in the failing heart. Circulation 94: 2837-2842, 1996.

78. Shao D, Oka S, Liu T, Zhai P, Ago T, Sciarretta S, Li H and Sadoshima J: A redox-dependent mechanism for regulation of AMPK activation by Thioredoxin1 during energy starvation. Cell Metab 19: 232-245, 2014.

79. Li YY, Chen D, Watkins SC and Feldman AM: Mitochondrial abnormalities in tumor necrosis factor-alpha-induced heart failure are associated with impaired DNA repair activity. Circulation 104: 2492-2497, 2001.

80. Hamblin M, Chang L, Fan Y, Zhang J and Chen YE: PPARs and the cardiovascular system. Antioxid Redox Signal 11: 1415-1452, 2009.

81. Gielen S, Schuler G and Adams V: Cardiovascular effects of exercise training: Molecular mechanisms. Circulation 122: 1221-1238, 2010

82. Holloway TM, Bloemberg D, da Silva ML, Simpson JA, Quadrilatero J and Spriet LL: High intensity interval and endurance training have opposing effects on markers of heart failure and cardiac remodeling in hypertensive rats. PLoS One 10: $\mathrm{e} 0121138,2015$.

83. Puhl SL, Müller A, Wagner M, Devaux Y, Böhm M, Wagner DR and Maack C: Exercise attenuates inflammation and limits scar thinning after myocardial infarction in mice. Am J Physiol Heart Circ Physiol 309: H345-H359, 2015.

84. Wisløff U, Loennechen JP, Falck G, Beisvag V, Currie S, Smith $G$ and Ellingsen O: Increased contractility and calcium sensitivity in cardiac myocytes isolated from endurance trained rats. Cardiovasc Res 50: 495-508, 2001.

85. Burniston JG: Adaptation of the rat cardiac proteome in response to intensity-controlled endurance exercise. Proteomics 9: 106-115, 2009. 
86. Rafalski K, Abdourahman A and Edwards JG: Early adaptations to training: Upregulation of alpha-myosin heavy chain gene expression. Med Sci Sports Exerc 39: 75-82, 2007.

87. Alves CR, da Cunha TF, da Paixão NA and Brum PC: Aerobic exercise training as therapy for cardiac and cancer cachexia. Life Sci 125: 9-14, 2015.

88. Gueritat J, Lefeuvre-Orfila L, Vincent S, Cretual A, Ravanat JL, Gratas-Delamarche A, Rannou-Bekono F and Rebillard A: Exercise training combined with antioxidant supplementation prevents the antiproliferative activity of their single treatment in prostate cancer through inhibition of redox adaptation. Free Radic Biol Med 77: 95-105, 2014.

89. Goh J, Tsai J, Bammler TK, Farin FM, Endicott E and Ladiges WC: Exercise training in transgenic mice is associated with attenuation of early breast cancer growth in a dose-dependent manner. PLoS One 8: e80123, 2013.

90. Deuster PA, Morrison SD and Ahrens RA: Endurance exercise modifies cachexia of tumor growth in rats. Med Sci Sports Exerc 17: 385-392, 1985

91. McGinnis GR, Ballmann C, Peters B, Nanayakkara G, Roberts M, Amin R and Quindry JC: Interleukin-6 mediates exercise preconditioning against myocardial ischemia reperfusion injury. Am J Physiol Heart Circ Physiol 308: H1423-H1433, 2015.

92. Petersen AM and Pedersen BK: The anti-inflammatory effect of exercise. J Appl Physiol 1985 98: 1154-1162, 2005

93. Rodriguez J, Fernández-Verdejo R, Pierre N, Priem F and Francaux M: Endurance training attenuates catabolic signals induced by TNF- $\alpha$ in muscle of mice. Med Sci Sports Exerc 48 227-234, 2016.

94. Gomes EC, Silva AN and de Oliveira MR: Oxidants, antioxidants, and the beneficial roles of exercise-induced production of reactive species. Oxid Med Cell Longev 2012: 756132, 2012.

95. Rebillard A, Lefeuvre-Orfila L, Gueritat J and Cillard J: Prostate cancer and physical activity: Adaptive response to oxidative stress. Free Radic Biol Med 60: 115-124, 2013.

96. Chicco AJ, Schneider CM and Hayward R: Voluntary exercise protects against acute doxorubicin cardiotoxicity in the isolated perfused rat heart. Am J Physiol Regul Integr Comp Physiol 289: R424-R431, 2005

97. Powers SK, Morton AB, Ahn B and Smuder AJ: Redox control of skeletal muscle atrophy. Free Radic Biol Med 98: 208-217, 2016.
98. Ellison GM, Waring CD, Vicinanza C and Torella D: Physiological cardiac remodelling in response to endurance exercise training: Cellular and molecular mechanisms. Heart 98: 5-10, 2012.

99. Constantinou C, Fontes de Oliveira CC, Mintzopoulos D, Busquets S, He J, Kesarwani M, Mindrinos M, Rahme LG, Argilés JM and Tzika AA: Nuclear magnetic resonance in conjunction with functional genomics suggests mitochondrial dysfunction in a murine model of cancer cachexia. Int J Mol Med 27: 15-24, 2011.

100. Coffey VG and Hawley JA: The molecular bases of training adaptation. Sports Med 37: 737-763, 2007.

101. Scarpulla RC: Transcriptional activators and coactivators in the nuclear control of mitochondrial function in mammalian cells. Gene 286: 81-89, 2002.

102. Iemitsu M, Miyauchi T, Maeda S, Tanabe T, Takanashi M Irukayama-Tomobe Y, Sakai S, Ohmori H, Matsuda M and Yamaguchi I: Aging-induced decrease in the PPAR-alpha level in hearts is improved by exercise training. Am J Physiol Heart Circ Physiol 283: H1750-H1760, 2002.

103. Coven DL, Hu X, Cong L, Bergeron R, Shulman GI, Hardie DG and Young LH: Physiological role of AMP-activated protein kinase in the heart: Graded activation during exercise. Am J Physiol Endocrinol Metab 285: E629-E636, 2003.

104. Kraniou GN, Cameron-Smith D and Hargreaves M: Acute exercise and GLUT4 expression in human skeletal muscle: Influence of exercise intensity. J Appl Physiol 1985 101: 934-937, 2006.

105. DeBosch B, Treskov I, Lupu TS, Weinheimer C, Kovacs A Courtois $\mathrm{M}$ and Muslin AJ: Akt1 is required for physiological cardiac growth. Circulation 113: 2097-2104, 2006.

106. McMullen JR: Role of insulin-like growth factor 1 and phosphoinositide 3-kinase in a setting of heart disease. Clin Exp Pharmacol Physiol 35: 349-354, 2008.

107. McMullen JR, Amirahmadi F, Woodcock EA, SchinkeBraun M, Bouwman RD, Hewitt KA, Mollica JP, Zhang L, Zhang Y, Shioi T, et al: Protective effects of exercise and phosphoinositide 3-kinase(p110alpha) signaling in dilated and hypertrophic cardiomyopathy. Proc Natl Acad Sci USA 104: 612-617, 2007.

108. Santini MP, Tsao L, Monassier L, Theodoropoulos C, Carter J, Lara-Pezzi E, Slonimsky E, Salimova E, Delafontaine P, Song YH, et al: Enhancing repair of the mammalian heart. Circ Res 100: 1732-1740, 2007. 